Cahiers $d u$ MONDE RUSSE

\section{Cahiers du monde russe}

Russie - Empire russe - Union soviétique et États indépendants

$47 / 3 \mid 2006$

La Russie au XVIII

\title{
French books with Catherine II's coat of arms from the former Hermitage Library
}

Sergey V. Korolev

\section{OpenEdition}

Journals

Édition électronique

URL : https://journals.openedition.org/monderusse/8846

DOI : $10.4000 /$ monderusse. 8846

ISSN : $1777-5388$

Éditeur

Éditions de l'EHESS

Édition imprimée

Date de publication : 23 septembre 2006

Pagination : 659-666

ISBN : 978-2-7132-2097-5

ISSN : $1252-6576$

Référence électronique

Sergey V. Korolev, "French books with Catherine II's coat of arms from the former Hermitage Library », Cahiers du monde russe [En ligne], 47/3 | 2006, mis en ligne le 01 janvier 2007, consulté le 02 septembre 2022. URL : http://journals.openedition.org/monderusse/8846 ; DOI : https://doi.org/ 10.4000/monderusse.8846 
chercher : repérer : avancer

Cet article est disponible en ligne à l'adresse :

http://www.cairn.info/article.php?ID REVUE=CMR\&ID NUMPUBLIE=CMR 473\&ID ARTICLE=CMR 473 0659

French books with Catherine II's coat of arms from the former Hermitage Library

par Sergey V. KOROLEV

\section{Editions de l'EHESS | Cahiers du monde russe}

2006/3 - Vol 47

ISSN 1252-6576 | ISBN 9782713220975 | pages 659 à 666

Pour citer cet article :

-Korolev S., French books with Catherine II's coat of arms from the former Hermitage Library, Cahiers du monde russe 2006/3, Vol 47, p. 659-666.

Distribution électronique Cairn pour les Editions de l'EHESS.

(C) Editions de l'EHESS. Tous droits réservés pour tous pays.

La reproduction ou représentation de cet article, notamment par photocopie, n'est autorisée que dans les limites des conditions générales d'utilisation du site ou, le cas échéant, des conditions générales de la licence souscrite par votre établissement. Toute autre reproduction ou représentation, en tout ou partie, sous quelque forme et de quelque manière que ce soit, est interdite sauf accord préalable et écrit de l'éditeur, en dehors des cas prévus par la législation en vigueur en France. Il est précisé que son stockage dans une base de données est également interdit. 


\section{FRENCH BOOKS WITH CATHERINE II'S COAT OF ARMS FROM THE FORMER HERMITAGE LIBRARY}

"On ne produit rien ici d'un peu important dont on ne soit tenté de lui faire hommage. Elle [Catherine II] est l'idole de tous ceux qui pensent."

Diderot à Falconet, 20/03/1771

Among the books transferred from the Hermitage library to the Imperial Public Library (now National Library of Russia, NLR) in the middle of the 19th century, special attention should be given to the books having the imperial coat of arms of the Catherine II's epoch on the covers. These copies should be studied in the context of the attention which was given by the French scientific community to the Russian Empress at this time.

It is necessary to note that authors (or editors of these works) inspired by the Denis Diderot's example, undoubtedly hoped to receive some preferences from Catherine II. This explains that senders went into certain expenses, ordering not only making of "de luxe" binding, but also manufacturing of special stamp, which was impressed in both binding covers. Since styled definitions of imperial coat of arms differ considerably in details on all described copies (except works by $\mathrm{d}^{\prime}$ Alembert), we can suppose, that stamps were manufactured for being used only once and were produced by different masters.

We would like to note also, that Catherine II knew, some of the authors of the books. Other three authors received preliminary recommendations from Diderot, the person the Empress trusted in Paris.

The presence of the described copies in the Hermitage Library doesn't mean that Catherine II acquainted herself with them personally. At the end of 1770 s the amount of books and manuscripts addressed to Catherine was so big, that Russian diplomats in Europe were prohibited from receiving non-official gifts.

It is interesting, that impressing of styled imperial coat of arms on books addressed to Catherine II, is apparently solely French tradition: we haven't found any similar copy, from German or English origin. 
The copies are described according to the following plan:

H: $\quad$ Press mark according to the Hermitage Library book-plate of the 1st half of the 19th century (bookcase..., shelf...,\#...).

$\mathrm{N}$ : Modern press mark NLR.

Short-title.

Honorary degrees of the author, if they are indicated on the title page.

B: Characteristic features of the binding.

Printed dedication to Catherine II (if such is present).

1) $\mathrm{H}: 23.4 .45$.

$\mathrm{N}:$ 16.67.8.7.

Alembert, [Jean Le Rond] d',

Mélanges de littérature, d'histoire et de philosophie, nouv. éd., t. I-IV, Amsterdam : Z. Chatelein \& fils, 1763, in- $8^{\circ}$.

B : Red morocco; smooth spine gilt with floral, star and leaf tools. Edges gilt. Triple gilt fillets round sides. Endpapers are decorated with scattered 6cornered golden stars.

2) $\mathrm{H}: 1.1 .8$.

$\mathrm{N}: 15.5 .5 .74$.

Alembert, [Jean Le Rond] d'.

Élémens de musique, théorique et pratique, suivant les principes de M. Rameau, $<\ldots>$, nouv. éd., Lyon : Jean-Marie Bruyset, 1762 , in- $8^{\circ}$, pl. gr.

B : The same as \#1, except for the green moire endpapers.

3) $\mathrm{H}: 1.5 .85$.

$\mathrm{N}: 5.11 .5 .112$.

Bailly, [Jean-Silvain].

Histoire de l'Astronomie ancienne, depuis son origine jusqu'a l'établissement de l'école d'Alexandrie, <...>, 2e éd. Paris: De Bure fils aîné, 1781, pl.gr.

B : Red morocco; edges gilt. Raised bands. Triple gilt fillets round sides. Dark blue endpapers.

4) $\mathrm{H}: 1.1 .114$.

$\mathrm{N}:$ 16.32.10.6a.

Bailly, [Jean-Silvian].

Lettres sur l'origine des sciences, et sur celle des peuples de l'Asie, adressées à M. de Voltaire, précédées de quelques Lettres de M. de Voltaire à l'auteur, Londres (Paris) : 1777 , in- $8^{\circ}$.

B : Crimson morocco; edges gilt. At center of spine compartments a fleurons. Dark blue endpapers. 
5) $\mathrm{H}: 3.4 .185$.

$\mathrm{N}: 33.4 .3 .15$.

Bernardin de Saint-Pierre, [Jaques Henri] ${ }^{1}$.

Voyage à l'Isle de France, à l'Isle de Bourbon, au Cap de Bonne-Espérance, \&c. Avec des Observations nouvelles sur la nature \& sur les hommes, par un Officier du Roi, t. I-II, Amsterdam (Paris) : Merlin, 1773 , in- $8^{\circ}$., fr. et pl. gr. par J. Moreau le jeune.

B : Red morocco; edges gilt. Smooth spine gilt with fleurons. Marbled endpapers.

6) $\mathrm{H}: 7.3 .64$

$\mathrm{N}: 33.5 .5 .3$.

\section{Bossu, [Jean Bernard].}

[capitaine dans les troupes de la Marine].

Nouveaux voyages aux Indes Occidentales, contenant une Relation des différens peuples qui habitent les environs du grand Fleuve Saint-Louis, appelé vulgairement le Mississipi, leur religion, leur gouvernement, leurs moeurs \& leur commerce, pt. I-II en 1 vol. Paris : Le Jay, 1768, in-12, fr. et pl. gr. par de St.Aubin.

B : Red morocco; edges gilt. Smooth spine gilt; triple gilt fillets round sides. Small two-headed eagles in the corners of the both sides. Marbled endpapers.

7) $\mathrm{H}: 4.8 .83$.

$\mathrm{N}:$ 12.33.1.146.

\section{Bury, Richard de.}

Histoire de la vie de Henri IV, Roi de France et de Navarre <...>, t. I-II, Paris : Didot 1'aîné, 1765 , in- $4^{\circ}$, portr. gr. par St. Aubin.

B : Parisian burgundy morocco. Gold tooled with a design in the "dentelle" style. Marbled endpapers.

8) $\mathrm{H}: 2.1 .4$

$\mathrm{N}: 10.23 .3 .45$.

\section{Chabrit, Pierre 2 .}

De la Monarchie françoise, ou de Ses loix, t. I, Bouillon: La Société Typographique, 1783 , in $-8^{\circ}$.

B : Red morocco; edges gilt. Smooth spine gilt. Redspeckled paper endpapers.

1. About Bernardin de Saint-Pierre and Catherine II see: Alexandre F. Stroev. Les Aventuriers des Lumières. P.: PUF, 1997.

2. Diderot wrote about the author in his letter to Catherine II from 25.08.1781. Diderot D. Correspondance,. P.: G. Roth, 1970. T. XV.p. 266-268. 
9) $\mathrm{H}: 1.6 .119$.

$\mathrm{N}: 10.7 .5 .492$.

Constitutions des treize État-unis de l'Amérique [Tr. par Louis-Alexandre Duc de La Rochefoucauld d’Enville], Philadelphe - Paris : Ph. - D. Pierres; Pissot père \& fils, 1783 , in $-4^{\circ}$.

B : Red morocco; edges gilt. Triple gilt fillets round sides. Dark blue endpapers.

10) $\mathrm{H}: 1.7 .185$.

$\mathrm{N}: 12.51 .2 .63$.

Courtalon, L'Abbé.

Atlas élémentaire où l'on voit sur des cartes et des tableaux relatifs à l'objet l'état actuel de la Constitution politique de l'Empire d'Allemagne <...>. Le tout composé et vérifié d'après les meilleurs cartes nationales, la Géographie de Mr. Büsching, [\&c.], Paris : Julien \& Boudet, 1774 , in- $4^{\circ}$, en grand papier, titre et cartes gr.

Label grave on the last endpaper : "Prix, broché $=15$ liv. / Relié en veau = 18 liv. / En gr. papier avec filets dorés = 24 liv."

B : Red morocco; edges gilt. Spine richly gilt with floral and leaf tools. Marbled endpapers.

11) $\mathrm{H}: 13.4 .57$.

$\mathrm{N}: 6.54 .4 .53$.

Darluc.

[professeur royal de médecine en l'université d'Aix, associé à l'Académie des Sciences \& Belles-Lettres de Marseille]

L'Inoculation victorieuse des préjugés et l'envie. Poème en douze chants avec des notes, Avignon : Piere-François Chabrier, 1773, in- $8^{\circ}$.

p. i - vi : Dedication to Catherine II.

B : Light-gray moire; edges gilt. Pink silk endpapers. Silver embroidered Russian imperial coat of arms in the middles of both sides.

12) $\mathrm{H}: 21.2 .27$.

$\mathrm{N}: 15.28 .7 .106$.

Ducarne de Blangy, [Jacques Joseph].

Traité de l'éducation économique des abeilles, où se trouve aussi leur histoire naturelle, pt. I-II en 1 vol., Paris : P.Fr. Gueffier, 1771, in-12, fig.

B : Red morocco; edges gilt. Spine decorated in compartments by floral design. Endpapers are decorated with scattered 6-cornered golden stars.

13) $\mathrm{H}: 17.2 .34$

$\mathrm{N}: 36.25 .9 .40$.

Épinay, [Louise-Florence-Pétronille Tardieu d'Esclavelles, Mise d' .]

Les Conversations d'Émilie, 5e éd., t. I-II, Paris : Belin, 1788, in-12, fr. gr par J. M. Moreau jr. 
Dedicated to Catherine II by Emilie de Belsunce, (“20 Février, 1786”.)

B : Green morocco; edges gilt. Spine with the tool of a harp in the center of compartments. Straight-crimson endpapers.

14) $\mathrm{H}: 25.2 .22$.

$\mathrm{N}:$ 10.19.7.200.

Graslin, [Jean Joseph Louis].

Essai analytique sur la richesse et sur l'impôt, où l'on réfute la nouvelle doctrine économique, qui a fourni à la Sociéte royale d'agriculture de Limoges les principes d'un Programme qu'elle a publié sur l'effet des Impôts inderects, Londres, 1767, in- $8^{\circ}$.

B : Red morocco; edges gilt. Triple gilt fillets round sides; small fleurons in the corner of the both covers.

15) $\mathrm{H}: 28.2 .27$

$\mathrm{N}: 5.8 .3 .83$.

Grignon, [Pierre Clément].

[maître de forge, correspondant d'Académie royale des Sciences, \& c.]

Mémoires de physique sur l'art de fabrique le fer; d'eu fondre \& forger des canons d'artillerie, sur l'histoire naturelle, et sur divers sujets particuliers de physique et $d^{\prime}$ économie, $<\ldots>$, Paris : Delalain, 1775 , in $-4^{\circ}$, pl. gr.

B : Red morocco; edges gilt. Each side having an interlaced geometrical border, within which is a panel formed of ornamentical tooling. Dark blue endpapers.

16) $\mathrm{H}: 1.3 .224$

$\mathrm{N}: 4.10 .5 .127$.

Jacquin, 1'abbé [Armand-Pierre].

chapelain des Mesdames Victoire \& Sophie; membre des Académies royales de Rouen \& de Metz; honoraire de la Société littéraire d'Arras]

De la Santé, ouvrage utile à tout le monde, 4 e éd., Paris : G.Desprez, 1771, in-12.

B : Red morocco; edges gilt. Smooth spine gilt. Endpapers are decorated with scattered 6-cornered golden stars.

17) $\mathrm{H}: 1.1 .6$.

$\mathrm{N}: 10.21 .6 .90$.

Landreau de Maine-au-Picq.

[avocat, \& asseseur de la Maréchaussee de Saintes]

Législation philosophique, politique et morale, t. I-III, Genève (Paris) : De Bure aîné, 1787 , in-12.

B : Red morocco; marbled edges. Endpapers gilt. 
18) $\mathrm{H}: 21.6 .67$.

$\mathrm{N}: 15.10 .4 .25$.

La Salle de L'Estang, [Simon Philibert] de ${ }^{3}$.

[seigneur de Muyr, Tinqueux, \&c., ancien député de la ville Rheims à Paris]

Manuel d'Agriculture pour le propriétaire et pour le gouvernement; <...>. Avec la Réfutation de la nouvelle Méthode de M. Thull, Paris : Lottin l'aîné; Dessain junior, 1764. in- $8^{\circ}$, fr. gr. par Cochin.

B : Green morocco; edges gilt. Triple gilt fillets round sides. At the center of spine compartments a small Russian imperial eagles. Endpapers are decorated with scattered 6-cornered golden stars.

19) $\mathrm{H}: 1.1 .43$.

$\mathrm{N}: 33.5 .5 .14$.

Le Cointe, [Jean-Louis] $]^{4}$.

[capitaine de cavalerie au régiment de Conty, de l'Académie royale de Nîmes]

Commentaires sur la "Retraite des Dix-mille" de Xénophon; à l'usage des jeunes officiers, t. I-II, Paris : Nyon; Saillant, 1766, in-12, carte gr.

B : Red morocco; edges gilt. Triple gilt fillets round sides. Marbled endpapers.

20) $\mathrm{H}: 2.1 .23$

$\mathrm{N}:$ 5.9.9.61.

Ibid.

La Science des postes militaires, ou Traité des fortifications de campagne, à l'usage des officiers particuliers d'Infanterie qiu sont détachés à la guerre, Paris : Desaint \& Saillant, 1759. in $-8^{\circ}$, pl. gr.

B : See \# 19.

21) $\mathrm{H}: 3.8 .15$.

$\mathrm{N}: 10.22 .1 .144$.

Le Mercie de la Riviere, [Pierre-Paul-Francois-Joachim-Henri $]^{5}$.

$L^{\prime}$ Ordre naturel et essentuel des sociétés politiques, Londres : Jean Nourse (Paris : Desaint), 1767 , in $-4^{\circ}$.

B : Crimson morocco; edges gilt. Triple gilt fillets round sides. Raised bands. Polished marbled endpapers.

There are MS (ink) corrections on the pp. $15 \& 361$

3. Catherine II mentioned the author in her letter to Voltaire from 10/21.06.1771 (Best. D.,17 256).

4. The books of Le Cointe were recommended to Catherine II by Diderot. See: Correspondance de Falconet avec Catherine II, 1767-1778, P.: L. Réau, 1921, p. 147-149.)

5. About Le Mercier de La Riviere and Catherine II see: Alexandre F. Stroev... 
22) $\mathrm{H}: 27.2 .21$

$\mathrm{N}:$ : 5.9.3.94.

Marat, [Jean-Paul].

[docteur en médecine \& médecin des gardes-du-corps de Monseigneur le Comte d'Artois]

Découvertes $<\ldots .>$ sur la lumière; constatées par une suite d'expériences nouvelles $<\ldots>$, Londres (Paris) : Jombert, fils aîné, 1780, in- $8^{\circ}$.

B : Red Morocco; edges gilt. Smooth spine richly gilt and decorated with geometrical figures. Marbled endpapers.

23) $\mathrm{H}: 27.2 .23$

$\mathrm{N}:$ 5.9.3.36.

Ibid.

Recherches physiques sur l'électricité, Paris : De l’Imprimerie de Clousier; Nyon, 1782, in- $8^{\circ}$, pl. gr.

B : Red morocco; edges gilt. Smooth spine gilt with stylized two-headed eagles at center of spine compartments. Dark blue endpapers.

24) $\mathrm{H}: 27.2 .22$.

$\mathrm{N}: 5.9 .3 .95$.

Ibid.

Recherches physiques sur le feu. Paris : Chez C. A. Jombert, fils aîné, 1780, in- $8^{\circ}$, pl.gr.

B : Red morocco; edges gilt. Triple gilt fillets round sides. Smooth spine gilt. Endpapers are decorated with scattered 6-cornered golden stars.

25) $\mathrm{H}: 16.8 .181$.

$\mathrm{N}: 6.56 .1 .12$.

Marmontel, [Jean-François].

Chefs-d'oeuvre dramatiques, ou Recueil des meilleures pièces du Théatre françois, tragique, comique et lirique; Avec des discours préliminaires sur les trois genres, \& des remarques sur la langue \& de gout, Paris : De 1’Imprimerie de Grange, 1773, in$4^{\circ}$, fig., vignettes et 10 culs-de-lampe par Eisen, gravés par N. de Launay \&c.

B : Red morocco; edges gilt. Gold tooled dentelle border with the small bird tool. Raised bands. Dark blue silk endpapers.

26) $\mathrm{H}: 1.8 .268$.

$\mathrm{N}: 10.3 .1 .39$.

Muyart de Vouglans, [Pierre-François].

Les Loix criminelles de France, dans leur ordre naturel, Paris : Mérigot le jeune, 1780 , in-fol ${ }^{\circ}$.

B : Red morocco; edges gilt. Raised bands. Spine gilt with floral, star and leaf tools. Dark-blue endpapers. 
$27 \mathrm{H}: 2.2 .33$.

$\mathrm{N}:$ 6.57.5.15.

Ormoy, [Charlotte Chaumet, Mme d'].

La Vertu chancelante, ou la Vie de Mlle d'Amicourt; Dediée au Roi de Prusse, Liége (Paris) : Moureau, 1778, in-12.

B : Red morocco; edges gilt. Triple gilt fillets round sides. Smooth spine gilt with scrolls and scallops tool at center. Dark blue endpapers.

28) $\mathrm{H}: 13.5 .79$.

$\mathrm{N}: 6.11 .7 .91$.

Roman, [1'abbé Jean-Joseph-Thérèse].

L'Inoculation, poème en quatre chants, par M.L.R, Amsterdam (Paris) : Lacombe, 1773 , in- $8^{\circ}$, pl.gr.

P. i-ix : Dedication to Catherine II.

B : Green morocco; edges gilt. Smooth spine gilt. Light pink silk endpapers. Label grave: "Relié par/Derome le Jeune/rue St Jacques/au dessins de St Benoît".

29) $\mathrm{H}: 4.3 .74$.

$\mathrm{N}: 5.22 .10 .238$.

Tellès d'Acosta, [Dominique-Antoine].

[gran-maître des eaux \& forêts de France, ancien intendant de feu Mme la Daphine] Instruction sur les bois de marine et autres $<\ldots>$, Paris : Vve Duchesne; Clousier, 1782 , in $-12^{\circ}$.

B : Red morocco; edges gilt. Triple gilt fillets round sides. Smooth spine gilt. Dark blue endpapers.

National Library of Russia.

bookart@yandex.ru 